\title{
Scaling Laws for Two-Dimensional Random Ad-Hoc Wireless Networks
}

\author{
Ayfer Özgür and Olivier Lévêque \\ Ecole Polythechnique Fédérale de Lausanne \\ IC-ISC-LTHI, Building INR, Station 14 \\ CH-1015 Lausanne, Switzerland \\ Email: \{Ayfer.Ozgur, Olivier.Leveque\}@epfl.ch
}

\begin{abstract}
We derive an information theoretic scaling law for the maximum achievable rate per communication pair in a twodimensional random ad-hoc wireless network. Our scaling law holds for non-absorptive media and when the path loss exponent (describing the decay of the amplitude of the signal) is between 1 and 2 . The key ingredient of our result is the recently established information theoretic scaling law for one-dimensional ad-hoc wireless networks in the attenuation regime of interest.
\end{abstract}

\section{INTRODUCTION}

The seminal work of P. Gupta and P. R. Kumar [1] on the capacity of ad hoc wireless networks implies that the maximum achievable rate per communication pair $R$ in a $d$ dimensional extended network with uniform traffic pattern, is bounded above by

$$
R \leq \frac{K}{n^{\frac{1}{d}}} .
$$

Recent attempts (see [2], [3], [4], [5]) have confirmed this result from information theoretic point of view for absorptive media or under strong assumptions on the attenuation. In the more interesting case when there is no absorption and when the path loss exponent is between 1 and 2, there is a gap between the results presented in [4] and the upper bound (1). This gap has been closed recently in [5] for one-dimensional networks. In this paper, using the result of [5], we derive an information theoretic upper bound on the maximum achievable rate per communication pair in a two-dimensional random adhoc wireless network which improves the already known upper bounds in the attenuation regime of interest.

Main Result: If the attenuation function describing the decay of the transmitted signals over distance is given by

$$
g(r)=\frac{1}{r^{\delta}}
$$

where $1 \leq \delta \leq 2$, then for all $\varepsilon>0$

$$
R \leq \frac{K}{n^{\frac{1}{2}-\frac{1}{2(\delta+4)}-\varepsilon}} \quad \text { a.s. }
$$

in a large uniformly distributed two-dimensional network, where $K>0$ is a constant independent of $n$.

Remark: It is not difficult to extend our result and show that the transport capacity of such a network is almost surely bounded above by

$$
C_{T} \leq K n^{1+\frac{1}{2(\delta+4)}+\varepsilon}
$$

which we will not prove here due to space limitations.

\section{APPROACH}

We consider a constant density network of $n$ users (or nodes) where the users are independently and uniformly distributed on the two-dimensional domain $D=[-\sqrt{n}, \sqrt{n}] \times$ $[0, \sqrt{n}]$. Let us divide this domain into two equal parts $[-\sqrt{n}, 0] \times[0, \sqrt{n}]$ and $[0, \sqrt{n}] \times[0, \sqrt{n}]$. We are interested in bounding the total information flow from one half of the network to the other, or equivalently the sum of the rates of communications passing the imaginary boundary on the $y$ axis, say from left to right.

Let us make a series of optimistic assumptions: in parallel to [4] and [5], we first introduce $n$ additional "mirror" users that help relaying traffic, where the mirror location of $\left(x_{i}, y_{i}\right)$ is $\left(-x_{i}, y_{i}\right)$. After the introduction of mirror users, there are exactly $n$ users on each side of the domain, symmetrically located with respect to the $y$-axis and moreover independently and uniformly distributed on each side. Let us also assume that the users on each side of the domain can share instantaneous information and power among themselves so that we are in the following MIMO channel setting,

$$
\boldsymbol{V}_{\boldsymbol{i}}=\sum_{j=1}^{n} G_{i j}^{\delta, n} \boldsymbol{U}_{j}+\boldsymbol{Z}_{\boldsymbol{i}}, \quad i=1, \ldots n
$$

where $\boldsymbol{Z}=\left(\boldsymbol{Z}_{\mathbf{1}}, \ldots, \boldsymbol{Z}_{\boldsymbol{n}}\right)$ is a vector of circularly symmetric complex Gaussian random variables with unit variance and the entries of the $n \times n$ channel matrix $G^{\delta, n}$ are given by

$$
G_{i j}^{\delta, n}=\frac{1}{\left(\left(x_{i}+x_{j}\right)^{2}+\left(y_{i}-y_{j}\right)^{2}\right)^{\frac{\delta}{2}}}
$$

where $\left(L_{i}:=\left(x_{i}, y_{i}\right), i=1, \ldots, n\right)$ are the right-hand side node locations. In the following sections, we will use the notation $G^{\delta, n}\left\{L_{1}, \ldots L_{n}\right\}$ to refer to the $n \times n$ matrix $G^{\delta, n}$ corresponding to a specific configuration $\left(L_{1}, \ldots L_{n}\right)$ or omit the argument when no confusion can arise. Under the power constraint $\sum_{j=1}^{n} E\left[\left|\boldsymbol{U}_{\boldsymbol{j}}\right|^{2}\right] \leq n P$, the capacity of the above channel upper bounds the maximum flow of information from one half of the network to the other and is given by

$$
C_{n}=\max _{P_{k} \geq 0: \sum_{k=1}^{n} P_{k} \leq n P} \sum_{k=1}^{n} \log \left(1+P_{k} \lambda_{k}^{2}\right)
$$

where $\lambda_{k}$ are the eigenvalues of the symmetric matrix $G^{\delta, n}$. Noting that $P_{k} \leq n P$ and $\lambda_{k} \geq 0$ for each $k$, we further 
obtain

$$
\begin{aligned}
C_{n} & \leq \sum_{k=1}^{n} \log \left(1+n P \lambda_{k}^{2}\right) \leq \sum_{k=1}^{n} \log \left(1+\sqrt{n P} \lambda_{k}\right)^{2} \\
& =2 \log \operatorname{det}\left(I+\sqrt{n P} G^{\delta, n}\right) .
\end{aligned}
$$

The proof of the fact that $G^{\delta, n}$ is non-negative definite can be found in [4].

If we assume a uniform traffic pattern, there will be order $n$ communication requests that need to pass the boundary from left to right which gives

$$
R \leq K \frac{C_{n}}{n}
$$

Hence, we are interested in determining the scaling of the sum-capacity $C_{n}$ in the above described setting.

In the one-dimensional case, the same approach leads us to the same upper bound (3) on $C_{n}$, however the structure of the $n \times n$ channel matrix $G^{1 D^{\delta, n}}$ is given by

$$
G_{i j}^{1 D^{\delta, n}}=\frac{1}{\left(x_{i}+x_{j}\right)^{\delta}}
$$

in this case. When the channel matrix has this simpler structure, it has been shown in [5] that $C_{n}$ is bounded above by

$$
C_{n} \leq 2 \log \operatorname{det}\left(I+\sqrt{n P} G^{1 D^{\delta, n}}\right) \leq K(\log n)^{3+\sigma}
$$

for $1 \leq \delta \leq 2$, where $K>0$ is a constant independent of $n$ and $\delta$, and $\sigma>0$ is arbitrarily small. The upper bound (5) is established for linear networks satisfying a minimum distance constraint, however the same bound holds for uniformly distributed large random networks in the almost sure sense.

\section{PROOF}

Our approach is to divide the planar network into horizontal strips and make use of the result obtained for linear networks. Hence, we start by dividing the domain $D$ into $N=\frac{\sqrt{n}}{\epsilon}$ equal horizontal strips, namely

$$
S_{i}=[-\sqrt{n}, \sqrt{n}] \times[(i-1) \epsilon, i \epsilon] \quad \text { for } \quad i=1,2 \ldots N .
$$

Let us denote the total number of users in the strip $S_{i}$ (with symmetric left and right-hand side configuration) by the random variable $2 \boldsymbol{n}_{\boldsymbol{i}}$. We recall the generalized Hadamard's Inequality (see [6], Thm 9.C.1): If $A^{(n)}$ is $n \times n$ Hermitian positive definite matrix and $\left(A^{\left(s_{i}\right)}, i=1, \ldots, p\right)$ are the diagonal blocks of $A$ of given sizes $\left\{s_{i}\right\}$ (such that $n=\sum_{i=1}^{p} s_{i}$ ) then

$$
\operatorname{det}(A) \leq \prod_{i=1}^{p} \operatorname{det}\left(A^{\left(s_{i}\right)}\right) .
$$

We can apply this inequality to the positive definite matrix $\left(I+\sqrt{n P} G^{\delta, n}\right)$ with the diagonal blocks being the $N$ strips we have introduced. Hence, we can bound the sum-capacity of the planar network by,

$$
\begin{aligned}
C_{n} & \leq 2 \log \operatorname{det}\left(I+\sqrt{n P} G^{\delta, n}\right) \\
& \leq \sum_{i=1}^{N} 2 \log \operatorname{det}\left(I+\sqrt{n P} G^{\delta, n_{i}}\left\{S_{i}\right\}\right) .
\end{aligned}
$$

where now we use $S_{i}$ to refer to the configuration of the strip $S_{i}$. Let us now consider the expected value of this upper bound over random node locations, thus

$$
\begin{gathered}
E_{\left(\boldsymbol{x}_{1}, \boldsymbol{y}_{1}\right), \ldots,\left(\boldsymbol{x}_{n}, \boldsymbol{y}_{n}\right)}\left[\log \operatorname{det}\left(I+\sqrt{n P} \boldsymbol{G}^{\delta, n}\right)\right] \\
\leq E_{\boldsymbol{n}_{1}, \ldots, \boldsymbol{n}_{\boldsymbol{N}}}\left[E_{\boldsymbol{X}\left(S_{1}\right), \ldots, \boldsymbol{X}\left(S_{n}\right) ; \boldsymbol{Y}\left(S_{1}\right), \ldots, \boldsymbol{Y}\left(S_{n}\right)}\right. \\
\left.\left[\sum_{i=1}^{N} \log \operatorname{det}\left(I+\sqrt{n P} G^{\delta, \boldsymbol{n}_{\boldsymbol{i}}}\left\{\boldsymbol{S}_{\boldsymbol{i}}\right\}\right)\right]\right] \\
=\sum_{i=1}^{N} E_{\boldsymbol{n}_{\boldsymbol{i}}}\left[E_{\boldsymbol{X}\left(S_{i}\right)}\left[E_{\boldsymbol{Y}\left(S_{i}\right)}\left[\log \operatorname{det}\left(I+\sqrt{n P} G^{\delta, \boldsymbol{n}_{\boldsymbol{i}}}\left\{\boldsymbol{S}_{\boldsymbol{i}}\right\}\right)\right]\right]\right]
\end{gathered}
$$

where the subscripts denote the variables with respect to which the expectation is performed and $\boldsymbol{X}\left(S_{i}\right)$ refers to the collection $\left(\boldsymbol{x}_{1}, \ldots, \boldsymbol{x}_{\boldsymbol{n}_{i}}\right)$ denoting the $x$-coordinates of the nodes in $S_{i}$ (and similarly for $\boldsymbol{Y}\left(S_{i}\right)$ ). It is easy to see that the terms in (9) governing different strips $S_{i}$ are equal. Without loss of generality, we concentrate on the strip $S_{1}$ with number of users $\boldsymbol{n}_{1}$ and configuration $\boldsymbol{S}_{\mathbf{1}}$. For notational convenience, we denote the matrix $G^{\delta, n_{1}}\left\{\boldsymbol{S}_{1}\right\}$ by $\boldsymbol{G}^{S, \delta}, \boldsymbol{X}\left(S_{1}\right)$ by $\boldsymbol{X}$ and $\boldsymbol{Y}\left(S_{1}\right)$ by $\boldsymbol{Y}$, however we keep in mind that the node locations $\left(\boldsymbol{x}_{\boldsymbol{i}}, \boldsymbol{y}_{\boldsymbol{i}}\right), 1 \leq i \leq \boldsymbol{n}_{\mathbf{1}}$ are now uniformly and independently distributed on the set $[0, \sqrt{n}] \times[0, \epsilon]$. Considering the inner most expectation for given $n_{1}$ and a set of $X$ and recalling that $\log \operatorname{det}(\cdot)$ is a concave function on the set of positive definite matrices, we apply Jensen's Inequality to obtain

$$
\begin{aligned}
E_{\boldsymbol{Y}} & {\left[\log \operatorname{det}\left(I+\sqrt{n P} \boldsymbol{G}^{S, \delta}\right)\right] } \\
& \leq \log \operatorname{det}\left(I+\sqrt{n P} E_{\boldsymbol{Y}}\left[\boldsymbol{G}^{S, \delta}\right]\right) .
\end{aligned}
$$

The entries of the matrix $\boldsymbol{G}^{S, \delta}$ are given by (2). Given $X$, each $\boldsymbol{y}_{i}, 1 \leq i \leq n_{1}$ is uniformly and independently distributed on the interval $[0, \epsilon]$ and the random variable $\boldsymbol{y}=\left(\boldsymbol{y}_{\boldsymbol{i}}-\boldsymbol{y}_{\boldsymbol{j}}\right)^{2}$ has a distribution $p_{\boldsymbol{y}}(y)$ supported on the interval $\left[0, \epsilon^{2}\right]$ when $i \neq j$. Thus the entries of the matrix $E_{\boldsymbol{Y}}\left[\boldsymbol{G}^{S, \delta}\right]$ are given by

$$
\begin{aligned}
E_{\boldsymbol{Y}}\left[\begin{array}{c}
\boldsymbol{G}_{i j}^{S, \delta} \\
i \neq j
\end{array}\right] & =\int_{0}^{\epsilon^{2}} p_{\boldsymbol{y}}(y) \frac{1}{\left(\left(x_{i}+x_{j}\right)^{2}+y\right)^{\frac{\delta}{2}}} d y \\
E_{\boldsymbol{Y}}\left[\boldsymbol{G}_{i i}^{S, \delta}\right] & =\frac{1}{\left(2 x_{i}\right)^{\delta}} .
\end{aligned}
$$

The matrix $E_{\boldsymbol{Y}}\left[\boldsymbol{G}^{S, \delta}\right]$ can be written as a sum of two matrices

$$
E_{\boldsymbol{Y}}\left[\boldsymbol{G}^{S, \delta}\right]={D^{\prime}}^{\delta}+G^{\prime^{\delta}}
$$

where $G^{\prime}$ is the matrix whose entries are given by

$$
G_{i j}^{\prime^{\delta}}=\int_{0}^{\epsilon^{2}} p_{\boldsymbol{y}}(y) \frac{1}{\left(\left(x_{i}+x_{j}\right)^{2}+y\right)^{\frac{\delta}{2}}} d y
$$

and ${D^{\prime}}^{\delta}$ is the diagonal matrix that compensates the difference between the diagonal entries of $G^{\prime}$ and $E_{\boldsymbol{Y}}\left[G^{S, \delta}\right]$. Thus,

$$
{D_{i i}^{\prime}}^{\delta}=\frac{1}{\left(2 x_{i}\right)^{\delta}} \int_{0}^{\epsilon^{2}} p_{\boldsymbol{y}}(y)\left(1-\left(1+\frac{y}{\left(2 x_{i}\right)^{2}}\right)^{-\frac{\delta}{2}}\right) d y .
$$


The entries of the diagonal matrix $D_{i i}^{\prime \delta}$ can be upper bounded by making use of the relation

$$
1-(1+x)^{-\alpha}=\int_{0}^{x} \alpha(1+z)^{-\alpha-1} d z \leq \alpha x
$$

which yields

$$
D_{i i}^{\prime} \leq \frac{\delta / 2}{\left(2 x_{i}\right)^{\delta+2}} \int_{0}^{\epsilon^{2}} y p_{\boldsymbol{y}}(y) d y \leq \frac{\delta \epsilon^{2}}{2\left(2 x_{i}\right)^{\delta+2}}=D_{i i}^{\delta}
$$

where $D^{\delta}$ is defined as the upper bounding diagonal matrix. In the appendix, we prove that the difference matrix $G^{1 D^{\delta}}-G^{\delta^{\delta}}$ whose entries are given by

$G_{i j}^{1 D^{\delta}}-G_{i j}^{\prime^{\delta}}=\frac{1}{\left(x_{i}+x_{j}\right)^{\delta}}-\int_{0}^{\epsilon^{2}} p_{\boldsymbol{y}}(y) \frac{1}{\left(\left(x_{i}+x_{j}\right)^{2}+y\right)^{\frac{\delta}{2}}} d y$.

is non-negative definite. This fact together with (12) implies that $G^{1 D^{\delta}}+D^{\delta}-G^{\prime^{\delta}}-{D^{\prime}}^{\delta}$ is a non-negative definite matrix. Recalling that the $\log \operatorname{det}(\cdot)$ is not only concave, but also increasing on the set of positive definite matrices (see [6], 16.E) gives

$$
\begin{aligned}
& \log \operatorname{det}\left(I+\sqrt{n P} E_{\boldsymbol{Y}}\left[\boldsymbol{G}^{S, \delta}\right]\right) \\
& \leq \log \operatorname{det}\left(I+\sqrt{n P} D^{\delta}+\sqrt{n P} G^{1 D^{\delta}}\right) \\
& \leq \log \operatorname{det}\left(I+\sqrt{n P} D^{\delta}\right)+\log \operatorname{det}\left(I+\sqrt{n P} G^{1 D^{\delta}}\right)
\end{aligned}
$$

where the last inequality is due to the following entropy relation for independent Gaussian vectors: $h(\boldsymbol{Y}+\boldsymbol{X}+\boldsymbol{Z})+$ $h(\boldsymbol{X}) \leq h(\boldsymbol{Y}+\boldsymbol{X})+h(\boldsymbol{X}+\boldsymbol{Z})$.

The second term in (13) resembles the upper bound (5) governing linear networks except that $G^{1 D^{\delta}}$ is now $n_{1} \times n_{1}$ with $n_{1} \leq n$. However, by the interlacing property of symmetric matrices (see [8, Thm 4.3.8]) the $n_{1}$ largest eigenvalues of the matrix $G^{1 D^{\delta, n}}$ that has $G^{1 D^{\delta, n_{1}}}$ as an upper left submatrix dominate the eigenvalues of $G^{1 D^{\delta, n_{1}}}$. Toghether with the fact that $I+\sqrt{n P} G^{\delta}$ has all its eigenvalues larger than 1 , this implies

$$
\log \operatorname{det}\left(I+\sqrt{n P} G^{1 D^{\delta, n_{1}}}\right) \leq K^{\prime \prime}(\log n)^{3+\sigma}
$$

almost surely for large $n$.

For the first term in (13), let us consider the expectation over $\boldsymbol{X}$, thus

$$
\begin{aligned}
E_{\boldsymbol{X}} & {\left[\log \operatorname{det}\left(I+\sqrt{n P} \boldsymbol{D}^{\delta}\right)\right] } \\
& =E_{\boldsymbol{X}}\left[\sum_{i=1}^{n_{1}} \log \left(1+\sqrt{n P} \frac{\delta \epsilon^{2}}{2\left(2 \boldsymbol{x}_{\boldsymbol{i}}\right)^{\delta+2}}\right)\right] \\
& =n_{1} \int_{0}^{\sqrt{n}} \frac{1}{\sqrt{n}} \log \left(1+\frac{\delta \sqrt{P}}{2} \frac{n^{\frac{1}{2}-2 \eta}}{(2 x)^{\delta+2}}\right) d x
\end{aligned}
$$

where (15) is obtained by choosing $\epsilon=n^{-\eta}$ with $\eta>0$. We have the following lemma from [4] (Lemma 2.2) which states that for any $C, p>0$ and $\alpha>1$, there exists a constant $K^{\prime}>0$ such that for all sufficiently large $n$,

$$
\int_{0}^{n} d x \log \left(1+\frac{C n^{p}}{x^{\alpha}}\right) \leq K^{\prime} n^{\frac{p}{\alpha} \wedge 1} \log n
$$

where $a \wedge b$ is minimum of $a$ and $b$. Applying this lemma to (15) and performing the last expectation in (9) with respect to $n_{1}$ yields

$$
E_{\boldsymbol{n}_{1}}\left[E_{\boldsymbol{X}}\left[\log \operatorname{det}\left(I+\sqrt{n P} \boldsymbol{D}^{\delta}\right)\right]\right] \leq \epsilon K^{\prime} n^{\frac{1-4 \eta}{2(\delta+2)}} \log n
$$

since the expected number of nodes in $S_{1}$ is $\frac{n}{N}$ (and $N=\frac{\sqrt{n}}{\epsilon}$ ).

Combining all the results we have obtained until now yields the following expectation result:

$$
\begin{aligned}
& E_{\left(\boldsymbol{x}_{\mathbf{1}}, \boldsymbol{y}_{1}\right), \ldots,\left(\boldsymbol{x}_{n}, \boldsymbol{y}_{n}\right)}\left[\log \operatorname{det}\left(I+\sqrt{n P} \boldsymbol{G}^{\delta, n}\right)\right] \\
& \quad \leq K^{\prime} \sqrt{n} n^{\frac{1-4 \eta}{2(\delta+2)}} \log n+K^{\prime \prime} \sqrt{n} n^{\eta}(\log n)^{3+\sigma} \\
& \quad \leq K \sqrt{n} n^{\frac{1}{2(\delta+4)}+\varepsilon}
\end{aligned}
$$

for all $\varepsilon>0$, by choosing $\eta=\frac{1}{2(\delta+4)}$.

There remains to prove that there is concentration around the expectation and that the sublinear behavior of this upper bound on sum-capacity takes place almost surely. Let us define $\Phi^{n}$ to be the following real valued function of node locations

$$
\Phi^{n}\left(L_{1}, \ldots, L_{n}\right):=\log \operatorname{det}\left(I+\sqrt{n P} G^{\delta, n}\left\{L_{1}, \ldots, L_{n}\right\}\right) .
$$

We set out to prove the following proposition.

Proposition 3.1: For any $\epsilon>0$, we have

$$
\lim _{n \rightarrow \infty} \frac{\left|\Phi^{n}\left(\boldsymbol{L}_{\mathbf{1}}, \ldots, \boldsymbol{L}_{\boldsymbol{n}}\right)-E\left[\Phi^{n}\left(\boldsymbol{L}_{\mathbf{1}}, \ldots, \boldsymbol{L}_{\boldsymbol{n}}\right)\right]\right|}{n^{\frac{1}{2}+\epsilon}}=0
$$

almost surely.

Before the proof of Proposition 3.1, we introduce a concentration inequality due to McDiarmid [8].

Theorem 3.1: Let $\left(\boldsymbol{L}_{\mathbf{1}}, \boldsymbol{L}_{\mathbf{2}}, \ldots, \boldsymbol{L}_{\boldsymbol{n}}\right)$ be a family of independent random variables with $\boldsymbol{L}_{\boldsymbol{k}}$ taking values in a set $A_{k}$ for each $k$. Suppose that the real-valued function $f$ defined on $\Pi A_{k}$ satisfies

$$
\sup _{L_{1}, \ldots, L_{n}, L_{k}^{\prime}}\left|f\left(L_{1}, \ldots, L_{n}\right)-f\left(L_{1}, \ldots, L_{k}^{\prime}, \ldots, L_{n}\right)\right| \leq c_{k} .
$$

Then, for any $t \geq 0$

$$
P\left(\left|f\left(\boldsymbol{L}_{\mathbf{1}}, \ldots, \boldsymbol{L}_{\boldsymbol{n}}\right)-E\left[f\left(\boldsymbol{L}_{\mathbf{1}}, \ldots, \boldsymbol{L}_{\boldsymbol{n}}\right)\right]\right| \geq t\right) \leq 2 e^{\frac{-2 t^{2}}{\sum c_{k}^{2}}} .
$$

The proof of Proposition 3.1 is based on applying Theorem 3.1 to the function $\Phi^{n}$. The crucial step is to properly bound the amount of change that occurs in the value of the function $\Phi^{n}$ due to a change in one of its parameters. Note, however, that $\Phi^{n}$ is unbounded since the $\boldsymbol{x}_{\boldsymbol{i}}$ 's can be arbitrarily close to zero which will make the corresponding diagonal elements of $G^{\delta, n}$ go to infinity. The problem can be overcome by showing that $\boldsymbol{x}_{\boldsymbol{i}}$ 's are all bounded away from zero with high probability as $n$ goes to infinity and that under the condition that they are all bounded away from zero, the amount $\Phi^{n}$ can be affected from a change in one of the node positions is bounded.

Let us fix $\mu>0$. The probability that any of the $\boldsymbol{x}_{\boldsymbol{i}}$ 's is smaller than $n^{-\left(\frac{1}{2}+\mu\right)}$ is bounded above by

$$
P\left(\boldsymbol{x}_{\min }<n^{-\left(\frac{1}{2}+\mu\right)}\right) \leq n P\left(\boldsymbol{x}_{1}<n^{-\left(\frac{1}{2}+\mu\right)}\right)=n^{-\mu},
$$


since $\boldsymbol{x}_{\boldsymbol{i}}$ 's are uniformly and independently distributed on $[0, \sqrt{n}]$. On the other hand, under the condition that $\boldsymbol{x}_{\boldsymbol{m i n}} \geq$ $n^{-\left(\frac{1}{2}+\mu\right)},\left(\boldsymbol{L}_{\mathbf{1}}, \boldsymbol{L}_{\mathbf{2}}, \ldots, \boldsymbol{L}_{\boldsymbol{n}}\right)$ is still a family of independent random variables where each $\boldsymbol{L}_{\boldsymbol{i}}$ is now uniformly distributed on the set $\left[n^{-\left(\frac{1}{2}+\mu\right)}, \sqrt{n}\right] \times[0, \sqrt{n}]$.

Conditioned on $x_{\min } \geq n^{-\left(\frac{1}{2}+\mu\right)}$, let $\left(L_{1}, \ldots, L_{n-1}, L_{n}\right)$ and $\left(L_{1}, \ldots, L_{n-1}, L_{n}^{\prime}\right)$ be two configurations that differ only in the last co-ordinate. Let $\Psi^{n-1}$ be defined as the following function of $n-1$ node locations:

$$
\begin{aligned}
& \Psi^{n-1}\left(L_{1}, \ldots, L_{n-1}\right) \\
&:=\log \operatorname{det}\left(I+\sqrt{n P} G^{\delta, n-1}\left\{L_{1}, \ldots, L_{n-1}\right\}\right) .
\end{aligned}
$$

Now, we consider the difference in the value of the function $\Phi^{n}$ for the two configurations

$$
\begin{aligned}
& \left|\Phi^{n}\left(L_{1}, \ldots, L_{n-1}, L_{n}\right)-\Phi^{n}\left(L_{1}, \ldots, L_{n-1}, L_{n}^{\prime}\right)\right| \\
& \quad \leq\left|\Phi^{n}\left(L_{1}, \ldots, L_{n-1}, L_{n}\right)-\Psi^{n-1}\left(L_{1}, \ldots, L_{n-1}\right)\right| \\
& \quad+\left|\Psi^{n-1}\left(L_{1}, \ldots, L_{n-1}\right)-\Phi^{n}\left(L_{1}, \ldots, L_{n-1}, L_{n}^{\prime}\right)\right| .
\end{aligned}
$$

Concentrating on the first term in (16), let $\lambda_{1} \leq \cdots \leq \lambda_{n}$ be the eigenvalues of the $n \times n$ symmetric matrix $I+$ $\sqrt{n P} G^{\delta, n}\left\{L_{1}, \ldots, L_{n-1}, L_{n}\right\}$ and $\hat{\lambda}_{1} \leq \cdots \leq \hat{\lambda}_{n-1}$ be the eigenvalues of the $n-1 \times n-1$ symmetric matrix $I+\sqrt{n P} G^{\delta, n-1}\left\{L_{1}, \ldots, L_{n-1}\right\}$. Note that by the interlacing property for symmetric matrices, we have

$$
1 \leq \lambda_{1} \leq \hat{\lambda}_{1} \leq \lambda_{2} \leq \cdots \leq \lambda_{n-1} \leq \hat{\lambda}_{n-1} \leq \lambda_{n}
$$

Expressing the functions $\Phi^{n}$ and $\Psi^{n-1}$ in terms of these eigenvalues and recalling that the logarithm function is monotonically increasing yields

$$
\begin{aligned}
\mid \Phi^{n}\left(L_{1}, \ldots, L_{n}\right)- & \Psi^{n-1}\left(L_{1}, \ldots, L_{n-1}\right) \mid \\
& =\left|\sum_{i=1}^{n} \log \lambda_{i}-\sum_{i=1}^{n-1} \log \hat{\lambda}_{i}\right| \\
& =\sum_{i=1}^{n} \log \lambda_{i}-\sum_{i=1}^{n-1} \log \hat{\lambda}_{i} \leq \log \lambda_{n} .
\end{aligned}
$$

The largest eigenvalue $\lambda_{n}$ of $I+\sqrt{n P} G^{\delta, n}$ can be bounded by the trace of the matrix and the condition $x_{\min } \geq n^{-\left(\frac{1}{2}+\mu\right)}$,

$$
\log \lambda_{n} \leq \log \sum_{i=1}^{n}\left(1+\frac{\sqrt{n P}}{\left(2 x_{i}\right)^{\delta}}\right) \leq c_{1}(\mu)+c_{2}(\mu) \log n
$$

where $c_{1}(\mu), c_{2}(\mu)>0$ are constants independent of $n$. The argument for the first term in (16) holds similarly for the second term. Furthermore, since the numbering of the nodes is arbitrary the same bound applies whenever the two configurations differ in a single node location, this single node being any of the $n$ nodes. We can therefore apply Theorem 3.1 and obtain

$$
\begin{gathered}
P\left(\left|\boldsymbol{\Phi}^{\boldsymbol{n}}-E\left[\boldsymbol{\Phi}^{\boldsymbol{n}}\right]\right| \geq \lambda n^{\frac{1}{2}+\epsilon}\right) \leq P\left(\boldsymbol{x}_{\boldsymbol{m i n}} \geq n^{-\left(\frac{1}{2}+\mu\right)}\right) \\
\quad+P\left(\left|\boldsymbol{\Phi}^{\boldsymbol{n}}-E\left[\boldsymbol{\Phi}^{\boldsymbol{n}}\right]\right| \geq \lambda n^{\frac{1}{2}+\epsilon} \mid \boldsymbol{x}_{\boldsymbol{m i n}} \geq n^{-\left(\frac{1}{2}+\mu\right)}\right) \\
\quad \leq n^{-\mu}+2 \exp \left(-\frac{2 \lambda^{2} n^{2 \epsilon}}{4\left(c_{1}(\mu)+c_{2}(\mu) \log n\right)^{2}}\right)
\end{gathered}
$$

for all $\lambda>0$. Choosing $\mu>1$ and considering the BorelCantelli lemma completes the proof.

\section{CONClusion}

We established an improved information theoretic upper bound on the maximum achievable rate per communication pair in a two-dimensional random ad-hoc wireless network when the medium is not absorptive and the attenuation is moderate.

\section{APPENDIX}

In order to prove that the matrix $G^{1 D^{\delta}}-G^{\prime}$ is non-negative definite, it is sufficient to show that

$$
G_{j k}^{1 D^{\delta}}-G_{j k}^{\prime \prime^{\delta}}=\frac{1}{\left(x_{j}+x_{k}\right)^{\delta}}-\frac{1}{\left(\left(x_{j}+x_{k}\right)^{2}+y\right)^{\frac{\delta}{2}}}
$$

is a non-negative definite matrix for each $y$, since $G^{1 D^{\delta}}-G^{\prime^{\delta}}$ is a convex combination of matrices of this type. The proof is straight forward when the following equivalent expression for the entries of $G^{\prime \prime}{ }^{\delta}$ is considered

$$
\begin{aligned}
G_{j k}^{\prime \prime^{\delta}}=\frac{2}{\pi \Gamma(\delta)} \int_{0}^{\infty} d t \int_{0}^{\infty} d \xi(\xi \sinh t)^{\delta-1} \\
e^{-\xi(\cosh t)\left(x_{j}+x_{k}\right)} \cos (\xi y)
\end{aligned}
$$

where $\Gamma$ is the Euler Gamma function. The expression is valid for $\delta>0$ and can be obtained by considering ([9], formulas I.2.7 and I.18.29) and ([10], formula 9.6.23). Noticing that the entries of $G^{1 D^{\delta}}$ are obtained by substituting $y=0$ in (17) yields

$$
\begin{aligned}
& \sum_{j, k=1}^{n}\left(G_{j k}^{1 D^{\delta}}-G_{j k}^{\prime \prime^{\delta}}\right) c_{j} c_{k}=\frac{2}{\pi \Gamma(\delta)} \int_{0}^{\infty} d t \int_{0}^{\infty} d \xi \\
& (\xi \sinh t)^{\delta-1}\left(\sum_{j=1}^{n} c_{j} e^{-\xi x_{j} \cosh t}\right)^{2}(1-\cos \xi y) \geq 0 .
\end{aligned}
$$

\section{REFERENCES}

[1] P. Gupta and P. R. Kumar, The Capacity of Wireless Networks, IEEE Trans. on Information Theory 42 (2), 2000, 388-404.

[2] L. -L. Xie and P. R. Kumar, A Network Information Theory for Wireless Communications: Scaling Laws and Optimal Operation, IEEE Trans. on Information Theory 50 (5), 2004, 748-767.

[3] A. Jovicic, S. R. Kulkarni and P. Viswanath, Upper Bounds to Transport Capacity of Wireless Networks, IEEE Trans. on Information Theory 50 (11), 2004, 2555-2565.

[4] O. Lévêque and E. Telatar, Information Theoretic Upper Bounds on the Capacity of Large, Extended Ad-Hoc Wireless Networks, IEEE Trans. on Information Theory 51 (3), March 2005.

[5] O. Lévêque and E. Preissmann, Scaling Laws for One-Dimensional AdHoc Wireless Networks, to appear in IEEE Trans. on Information Theory, Nov 2005.

[6] A. W. Marshall and I. Olkin, Inequalities: Theory of Majorization and its Applications, Academic Press, 1979.

[7] R. A. Horn and C. R. Johson, Matrix Analysis, Cambridge University Press, 1985.

[8] M. Habib, C. McDiarmid, J. Ramizez-Alfonsin and B. Reed, Probabilistic Methods for Algorithmic Discrete Mathematics, Springer, 1998.

[9] F. Oberhettinger, Tables of Fourier Transforms and Fourier Transforms of Distributions, Berlin: Springer-Verlag, 1990.

[10] M. Abramowitz and I. A. Stegun, Handbook of Mathematical Functions, Washington: National Bureau of Standards, 1966. 